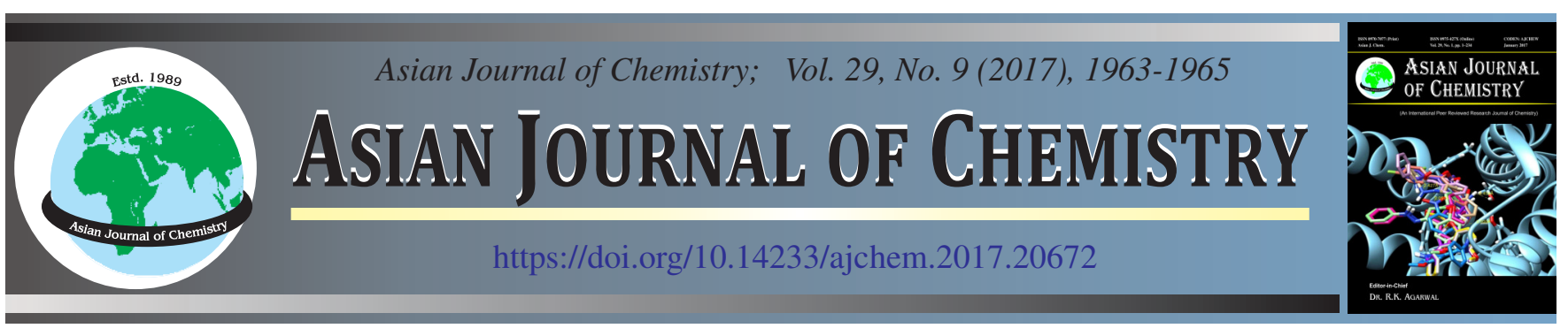

\title{
A Facile Synthesis of $\alpha$-Hydrazino Ketones from 1,3-Dicarbonyl Compounds Using 1,8-Diazobicyclo[5.4.0]undec-7-ene (DBU) as Organic Catalyst
}

\author{
R. Rejithamol ${ }^{*}$, K. Aparna, S. Swetha, A. Gayathri and S. Jisha
}

Department of Chemistry, Amrita School of Arts and Sciences, Amritapuri Campus, Amrita Vishwa Vidyapeetham University, P.O. Clappana, Kollam-690 525, India

*Corresponding author: E-mail: rejithamol01@gmail.com

Received: 27 March 2017;

Accepted: 11 May 2017;

Published online: 15 July 2017;

AJC-18474

A convenient and rapid method for the synthesis of $\alpha$-hydrazino ketones from 1,3-dicarbonyl compounds and diethyl azodicarboxylate in presence of 1,8-diazobicyclo[5.4.0]undec-7-ene as an organic catalyst at room temperature giving good yields, short reaction time and easy isolation.

Keywords: $\alpha$-Hydrazino ketone, 1,3-Dicarbonyl compounds, $\alpha$-Amination.

\section{INTRODUCTION}

Carbon-carbon and carbon-heteroatom bond-forming reactions are main interest to organic synthesis. Different types of reactions such as polar, pericyclic and radical reactions have been employed by organic chemists for the construction of carbon-carbon and carbon-heteroatom bonds. Amination is the process by which an amino group is introduced into an organic molecule. Most commonly, amination reactions involve the use of the amine as the nucleophile and the organic compound as the electrophile [1]. However, this sense of reactivity may be reversed for some electron-deficient amines, including oxaziridines, hydroxylamines, oximes and other $\mathrm{N}-\mathrm{O}$ substrates. Electrophilic amination [2] involving the formation of a carbon-nitrogen bond through the reaction of a nucleophilic carbanion with an electrophilic source of nitrogen $[3,4]$.

The electrophilic $\alpha$-amination of carbonyl compounds is widely used for the preparation of natural or unnatural $\alpha$-amino acids and $\alpha$-amino alcohols [5]. Aminating agents such as azidodicarboxylates [6], nitroso compounds or oxaziridines are typically used as electrophilic nitrogen source [7]. The addition of $\beta$-keto esters to azodicarboxylates is one of the preferred method for electrophilic amination [8,9]. Herein we present the addition of azodicarboxylates to 1,3-dicarbonyl compounds and $\beta$-keto esters in presence of the simple organic catalyst, 1,8-diazobicyclo[5.4.0]undec-7-ene (DBU).

\section{EXPERIMENTAL}

Melting points were recorded on a Büchi melting point apparatus and are uncorrected. NMR spectra were recorded at
$400 \mathrm{MHz}\left({ }^{1} \mathrm{H}\right)$ and $100 \mathrm{MHz}\left({ }^{13} \mathrm{C}\right)$ respectively on a Brüker Advance DPX-400 MHz NMR spectrometer. Chemical shifts are reported (d) relative to TMS $\left({ }^{1} \mathrm{H}\right)$ and $\mathrm{CDCl}_{3}\left({ }^{13} \mathrm{C}\right)$ as the internal standards. Coupling constants $(J)$ are reported in Hertz (Hz). IR spectra were recorded on Perkin Elmer FT-IR spectrophotometer. Commercial grade solvents were distilled prior to use.

General procedure for the synthesis of $\alpha$-hydrazino ketones from 3a-3f: A solution of appropriate 1,3-dicarbonyl compounds (1, $1 \mathrm{mmol})$ and DBU $(10 \mathrm{~mol} \%)$ in anhydrous $\mathrm{CH}_{2} \mathrm{Cl}_{2}(5 \mathrm{~mL})$ was stirred. To the solution diethyl azodicarboxylate $(2,1 \mathrm{mmol})$ was added and the reaction mixture stirred for $5 \mathrm{~min}$ at room temperature. The solvent was then removed by distillation under vacuum and the residue was subjected to column chromatographic separation (silica, hexane-ethyl acetate, 80:20).

Diethyl-1-(4,4-dimethyl-2,6-dioxocyclohexyl)hydrazine1,2-dicarboxylate (3a): Colourless viscous liquid, IR ( $\mathrm{KBr}$, $\left.V_{\max }, \mathrm{cm}^{-1}\right): 3436.4,3265.3,2088.2,1758.2,1694.2,1632.7$, 1026.7, 778.7. ${ }^{1} \mathrm{H}$ NMR (400 MHz, $\left.\mathrm{CDCl}_{3}\right): \delta 1.25-1.30(\mathrm{~m}$, $6 \mathrm{H}), 2.23(\mathrm{~s}, 6 \mathrm{H}), 4.18-4.27(\mathrm{~m}, 8 \mathrm{H}), 6.82(\mathrm{~s}, 1 \mathrm{H}), 7.09(\mathrm{~s}$, 1H) ppm. ${ }^{13} \mathrm{C}$ NMR $\left(100 \mathrm{MHz}, \mathrm{CDCl}_{3}\right): \delta 14.5,22.1,61.2$, 62.3, 63.6, 117.9, 155.8, 156.3, 191.9 ppm. HRMS: $m / z: 315$ [Calcd. for $\mathrm{C}_{14} \mathrm{H}_{22} \mathrm{~N}_{2} \mathrm{O}_{6}$ (314.15).

Diethyl-1-(1,3-dioxo-2,3-dihydro-1H-inden-2-yl)hydrazine-1,2-dicarboxylate (3b): Violet coloured solid, m.p.: 120 ${ }^{\circ} \mathrm{C}$, IR (KBr, $\left.v_{\max }, \mathrm{cm}^{-1}\right)$ : 3433, 2980, 2060, 1718,1630, 1230, 1100, 780. ${ }^{1} \mathrm{H}$ NMR $\left(400 \mathrm{MHz}, \mathrm{CDCl}_{3}\right): \delta 1.05-1.25(\mathrm{~m}, 6 \mathrm{H})$, 3.99-4.16 (m, 4H), 7.76-7.98 (m, 6H) ppm. ${ }^{13} \mathrm{C} \mathrm{NMR}(75 \mathrm{MHz}$ $\left.\mathrm{CDCl}_{3}\right): \delta 13.8,13.9,14.3,22.7,61.2,61.6,63.1,107.5,154.7$, 
155.9, 169.6, $194.6 \mathrm{ppm}$. HRMS: $\mathrm{m} / z$ : 321 [Calcd. for $\mathrm{C}_{15} \mathrm{H}_{16} \mathrm{~N}_{2} \mathrm{O}_{6}$ (320.10).

Diethyl-1-(4-chloro-1-ethoxy-1,3-dioxobutan-2-yl)hydrazine-1,2-dicarboxylate (3c): Yellow solid, m.p.: $80^{\circ} \mathrm{C}$, IR $\left(\mathrm{KBr}, \mathrm{v}_{\max }, \mathrm{cm}^{-1}\right)$ : v 3280, 3008, 2952, 2384, 1752, 1712, 1664, 1504, 1320, 1216, 1064, 768. ' H NMR (400 MHz, $\left.\mathrm{CDCl}_{3}\right): \delta 1.20-1.40(\mathrm{~m}, 9 \mathrm{H}), 4.10-4.40(\mathrm{~m}, 8 \mathrm{H}), 6.70(\mathrm{~s}, 1 \mathrm{H})$, $12.0(\mathrm{~s}, 1 \mathrm{H}) \mathrm{ppm} .{ }^{13} \mathrm{C} \mathrm{NMR}\left(100 \mathrm{MHz}, \mathrm{CDCl}_{3}\right): \delta 13.9,14.1$, 14.3, 38.8, 61.9, 62.2, 63.5, 108.0, 156.0, 169.2, 170.8, 197.1 ppm. HRMS: $m / z$ : 339 [Calcd. for $\mathrm{C}_{12} \mathrm{H}_{19} \mathrm{ClN}_{2} \mathrm{O}_{7}$ (338.09).

Diethyl-1-(2,6-dioxocyclohexyl)hydrazine-1,2-dicarboxylate (3d): Pale yellow liquid, IR $\left(\mathrm{KBr}, \nu_{\max }, \mathrm{cm}^{-1}\right)$ : 3392 , 2992, 2376, 1728, 1496, 1376, 1240, 1064, 784. ${ }^{1} \mathrm{H}$ NMR (400 MHz, $\left.\mathrm{CDCl}_{3}\right): \delta 1.21-1.25(\mathrm{~m}, 6 \mathrm{H}), 1.94-2.00(\mathrm{~m}, 4 \mathrm{H})$, $5.26(\mathrm{~s}, 1 \mathrm{H}), 8.24(\mathrm{~s}, 1 \mathrm{H}) \mathrm{ppm} .{ }^{13} \mathrm{C} \mathrm{NMR}\left(100 \mathrm{MHz}, \mathrm{CDCl}_{3}\right)$ : $\delta 13.8,13.9,14.3,22.7,61.2,61.6,63.1,107.5,154.7,155.9$, 169.6, 194.6 ppm. HRMS: m/z: 287 [Calcd. for $\mathrm{C}_{12} \mathrm{H}_{18} \mathrm{~N}_{2} \mathrm{O}_{6}$ (286.12)].

Diethyl-1-(1-ethoxy-1,3-dioxobutan-2-yl)hydrazine1,2-dicarboxylate(3e): Colourless Solid, m.p.: $82^{\circ} \mathrm{C}$, IR (KBr, $\left.v_{\max }, \mathrm{cm}^{-1}\right): \mathrm{v} 3392,2992,2376,1728,1496,1376,1240,1064$, 784. ${ }^{1} \mathrm{H}$ NMR (400 MHz, $\mathrm{CDCl}_{3}$ ): $\delta 1.25-1.45(\mathrm{~m}, 9 \mathrm{H}), 2.25$ (s, 3H), 4.13-4.36 (m, 6H), $6.75(\mathrm{~s}, 1 \mathrm{H}), 12.10(\mathrm{~s}, 1 \mathrm{H}) \mathrm{ppm}$. ${ }^{13} \mathrm{C} \mathrm{NMR}\left(100 \mathrm{MHz}, \mathrm{CDCl}_{3}\right): \delta 13.8,13.9,14.3,22.7,61.2$, 61.6, 63.1, 107.5, 154.7, 155.9, 169.6, 194.6 ppm. HRMS: $m / z: 305$ [Calcd. for $\mathrm{C}_{12} \mathrm{H}_{20} \mathrm{~N}_{2} \mathrm{O}_{7}$ (304.13)].

Diethyl-1-(1-ethoxy-1,3-dioxo-3-phenylpropan-2yl)hydrazine-1,2-dicarboxylate (3f): Pale yellow liquid, IR $\left(\mathrm{KBr}, \mathrm{v}_{\max }, \mathrm{cm}^{-1}\right)$ : 3433.2, 2987.9, 2063.5, 1714.9, 1633.8, 1238.7, 1188.9, 765.9. ${ }^{1} \mathrm{H}$ NMR (400 MHz, $\left.\mathrm{CDCl}_{3}\right): \delta 1.19-$ $1.33(\mathrm{~m}, 9 \mathrm{H}), 4.26-4.35(\mathrm{~m}, 6 \mathrm{H}), 6.50(\mathrm{~s}, 1 \mathrm{H}), 7.16(\mathrm{~s}, 1 \mathrm{H}$,$) ,$ $7.50(\mathrm{t}, J=7.6 \mathrm{~Hz}, 2 \mathrm{H}), 7.62(\mathrm{t}, J=8.8 \mathrm{~Hz}, 1 \mathrm{H}), 8.06(\mathrm{~d}, J=$ $7.6 \mathrm{~Hz}, 2 \mathrm{H}) \mathrm{ppm} .{ }^{13} \mathrm{C} \mathrm{NMR}\left(100 \mathrm{MHz}, \mathrm{CDCl}_{3}\right): \delta 13.8,13.9$, 14.3, 22.7, 61.2, 61.6, 63.1, 107.5, 154.7, 155.9, 169.6, 194.6 ppm. HRMS: m/z: 367 [Calcd. for $\mathrm{C}_{17} \mathrm{H}_{22} \mathrm{~N}_{2} \mathrm{O}_{7}$ (366.14)].

\section{RESULTS AND DISCUSSION}

Although many synthetic routes are employed for the synthesis of $\alpha$-aminated compounds, we wish to describe our results on the one pot synthesis of $\alpha$-hydrazino ketones from 1,3-dicarbonyl compounds using the simple organic catalyst 1,8-diazobicyclo[5.4.0] undec-7-ene (DBU). The treatment of 5,5'-dimethyl-1,3-cyclohexanedione (1) with diethyl azodicarboxy-late (2) (DEAD) in the presence of DBU under mild reaction conditions afforded the corres-ponding $\alpha$-hydrazino ester 3a in $98 \%$ yield (Scheme-I). Like 1,3-diketones, cyclic and acyclic $\beta$-keto esters reacted smoothly with diethyl azodicarboxylate under similar conditions to afford the respective $\alpha$-aminated esters (Table-1, entries $\mathbf{3 b}-\mathbf{f}$ ).
TABLE-1

REACTIONS BETWEEN 1,3-DICARBONYL COMPOUNDS AND DIETHYL AZODICARBOXYLATE

Entry

A mechanistic rationalization for this reaction is given in Scheme-II. It is conceivable that the initial event involved the abstraction of active methylene group proton from 1,3-dicarbonyl compound by DBU. It then adds to azadicarboxylate to afford an anionic intermediate, which followed by an intake of hydrogen ion afforded the desired product.<smiles>CCOC(=O)NN(C(=O)OCC)C1C(=O)CC(C)(C)CC1=O</smiles><smiles>CCOC(=O)N=NC(=O)OCC</smiles>

Scheme-II

\section{Conclusion}

We have unveiled a convenient one pot strategy for the synthesis of functionalized $\alpha$-hydrazino ketones from 1,3dicarbonyl compounds and diethyl azodicarboxylate in presence of 1,8-diazobicyclo[5.4.0] undec-7-ene (DBU) as an organic catalyst under mild reaction conditions.<smiles>CC1(C)CC(=O)CC(=O)C1</smiles>

1<smiles>CCOC(=O)N=NC(=O)OCC</smiles>

2<smiles>CCOC(=O)NN(C(=O)OCC)C1C(=O)CC(C)(C)CC1=O</smiles>

$\mathrm{CH}_{2} \mathrm{Cl}_{2}, 5 \mathrm{~m}, 98 \%$ 


\section{ACKNOWLEDGEMENTS}

The authors thank Sophisticated Test \& Instrumentation Centre, Cochin University of Science and Technology, Kochi, India for the characterization of the synthesized compounds.

\section{REFERENCES}

1. R.M. Williams, Synthesis of Optically Active Amino Acids; Pergamon Press: Oxford, pp. 167-185 (1989).

2. J. Yu, S.-S. Liu, J. Cui, X.-S. Hou and C. Zhang, Org. Lett., 14, 832 (2012);

https://doi.org/10.1021/o1203358f.

3. K. Juhl and K.A. Jørgensen, J. Am. Chem. Soc., 124, 2420 (2002); https://doi.org/10.1021/ja0175486.
4. M. Moreno-Mañas, J. Marquet and A. Vallribera, Tetrahedron, 52, 3377 (1996); https://doi.org/10.1016/0040-4020(95)01020-3.

5. B. Betzemeier and P. Knochel, Angew. Chem. Int. Ed. Engl., 36, 2623 (1997);

https://doi.org/10.1002/anie.199726231.

6. R.M. Williams and J.A. Hendrix, Chem. Rev., 92, 889 (1992); https://doi.org/10.1021/cr00013a007.

7. R.O. Duthaler, Angew. Chem. Int. Ed., 42, 975 (2003); https://doi.org/10.1002/anie.200390283.

8. M. Marigo, K. Juhl and K.A. Jorgensen, Angew. Chem. Int. Ed., 42, 1367 (2003);

https://doi.org/10.1002/anie.200390350.

9. J.S. Yadav, B.V.S. Reddy, C. Venugopal and B. Padmavani, Tetrahedron Lett., 45, 7507 (2004); https://doi.org/10.1016/j.tetlet.2004.07.156. 